Nota científica

\title{
Hypoglossum hypoglossoides (Delesseriaceae: Rhodophyta): un registro nuevo para la costa atlántica de México
}

\author{
Hypoglossum hypoglossoides (Delesseriaceae: Rhodophyta): a new record for the Atlantic \\ coast of Mexico
}

\author{
José Luis Godínez-Ortega ${ }^{\mathrm{a}, *}$, Pedro Ramírez-García ${ }^{\mathrm{a}}$, Alejandro Granados-Barba ${ }^{\mathrm{b}}$ \\ y Angélica Vázquez-Machorro ${ }^{c}$
}

\footnotetext{
a Departamento de Botánica, Instituto de Biología, Universidad Nacional Autónoma de México. Apartado postal 70-233, 04510 México, D. F., México

b Instituto de Ciencias Marinas y Pesquerías, Universidad Veracruzana. Calle Hidalgo 617, Col. Río Jamapa, 94290 Boca del Río, Veracruz, México

${ }^{\mathrm{c}}$ Posgrado en Ciencias del Mar y Limnología, Universidad Nacional Autónoma de México. Circuito Exterior, 04510 México, D. F., México
}

Recibido el 25 de febrero de 2015; aceptado el 10 de julio de 2015

Disponible en Internet el 7 de noviembre de 2015

\section{Resumen}

Se describen las características morfológicas de Hypoglossum hypoglossoides, un nuevo registro para la costa atlántica de México. Esta especie está presente en el Pecio Ana Elena del arrecife Anegada de Afuera, estado de Veracruz, golfo de México, de cuyo hábitat y distribución geográfica se proporciona información.

Derechos Reservados (C) 2015 Universidad Nacional Autónoma de México, Instituto de Biología. Este es un artículo de acceso abierto distribuido bajo los términos de la Licencia Creative Commons CC BY-NC-ND 4.0.

Palabras clave: Ceramiales; Pecio; Distribución geográfica

\section{Abstract}

The morphological characteristics of Hypoglossum hypoglossoides, a new record for the Atlantic coast of Mexico are described. This species was found in the Ana Elena shipwreck, Anegada de Afuera Reef, state of Veracruz, Gulf of Mexico, and information about its habitat and geographic distribution is provided.

All Rights Reserved (C) 2015 Universidad Nacional Autónoma de México, Instituto de Biología. This is an open access item distributed under the Creative Commons CC License BY-NC-ND 4.0.

Keywords: Ceramiales; Shipwreck; Geographical distribution

El Sistema Arrecifal Veracruzano (SAV) ha brindado abrigo al puerto de Veracruz desde su fundación (Valadez-Rocha y Ortiz-Lozano, 2013); no obstante, ha estado sujeto a encallamientos de embarcaciones desde la llegada de los españoles a América, por lo que es común la presencia de pecios en sus fondos. De acuerdo con Hayasaka-Ramírez y Ortiz-Lozano (2014), en el siglo pasado, al menos 126 embarcaciones encallaron en el SAV y, desde entonces, sobre éstas se llevan a cabo procesos

\footnotetext{
* Autor para correspondencia.

Correo electrónico: jlgo@unam.mx (J.L. Godínez-Ortega).

La revisión por pares es responsabilidad de la Universidad Nacional Autónoma de México.
}

de colonización y reclutamiento de organismos que son prácticamente desconocidos (Salas-Pérez y Granados-Barba, 2008). Este es el caso del pecio Ana Elena que lleva más de 60 años en el fondo marino y cuya biota dominante son afloramientos de comunidades incrustantes (Corallinales) que permiten el asentamiento de diversas especies algales comunes de los géneros Lithophyllum, Peyssonnelia, Sporolithon, Lobophora, entre otros; sin embargo, también se han encontrado especies raras y no registradas para la flora de México como la que se describe a continuación.

El género Hypoglossum Kützing, basado en Fucus hypoglossoides Stackhouse, perteneciente al orden Ceramiales, familia Delesseriaceae (Lin, Fredericq y Hommersand, 2001), está 
representado mundialmente por 30 especies, principalmente de aguas cálidas y templadas. Se caracteriza por presentar talo laminar monostromático con una vena axial, y tiene la característica de presentar hileras de células hasta del tercer orden (Wynne y Ballantine, 1986).

Según Wynne (2011), en la costa occidental del océano Atlántico, se han documentado 9 especies de Hypoglossum: $H$. anomalum M. J. Wynne et D. L. Ballantine, $H$. barbatum Okamura, $H$. caloglossoides M. J. Wynne et Kraft, H. hypoglossoides (Stackhouse) F. S. Collins et Hervey, H. involvens (Harvey) J. Agardh, H. rhizophorum D. L. Ballantine et M. J. Wynne, $H$. simulans M. J. Wynne, I. R. Price et D. L. Ballantine, H. subsimplex M. J. Wynne y H. tenuifolium (Harvey) J. Agardh. En México el género está representado únicamente por H. tenuifolium (Ortega, Godínez y Garduño-Solórzano, 2001), por lo que en este trabajo se cita por primera vez la presencia de $H$. hypoglossoides recolectada en el pecio Ana Elena situado en el arrecife anegada de afuera, Veracruz, México. En 3 salidas al campo realizadas al pecio $\left(19^{\circ} 10^{\prime} 07.76^{\prime \prime} \mathrm{N}\right.$, 95 51'39.12” O), el 21 marzo (NI-1779), 27 mayo de 2014 $(\mathrm{NI}-1683, \mathrm{MEXU}=2287)$ y 21 febrero $2015(\mathrm{NI}-1781)$, se recolectaron ejemplares usando equipo de buceo autónomo (SCUBA), ya que el barco se encuentra entre los 5 y los $12 \mathrm{~m}$ de profundidad. Las muestras se preservaron en una solución de formol neutralizado al $4 \%$ en agua de mar y otras fueron herborizadas. Para las observaciones morfoanatómicas se realizaron cortes a $20 \mu \mathrm{m}$ de espesor, los fragmentos fueron teñidos con una solución de azul de anilina al $1 \%$ y montados en miel karo-fenol 70/3\% (Tsuda y Abbott, 1985). Para la identificación del material se utilizó bibliografía especializada (Horta, Yokoya, Guimarães, Bacci y Oliveira, 2003; Littler y Littler, 2000; Oliveira-Filho, 1969; Womersley y Shepley, 1982; Wynne y Ballantine, 1986). Los ejemplares fueron depositados en el Herbario Nacional de México (MEXU).

Hypoglossum hypoglossoides (Stackhouse) F. S. Collins y Hervey, 1917, p. 116 (figs. 1-3)

Basónimo: Fucus hypoglossoides Stackhouse.

Localidad lectotipo: Polridmouth Cove, Cornwall, Inglaterra (Wynne, 1984).

\section{Diagnosis}

Talos laminares, postrados, delicados, color rosado pálido, de $1-3 \mathrm{~cm}$ de alto. Las hojas son lineales u ovales, $1-2 \mathrm{~cm}$ de largo y 1-2.5 mm de ancho, monostromáticas (fig. 1A); ramificación irregular que surge del eje central (fig. 1B); márgenes lisos a ondulantes; el eje central de una célula de 8-10 $\mu \mathrm{m}$ de ancho, con 2 hileras de células pericentrales laterales cilíndricas de 40$65 \mu \mathrm{m}$ de ancho y de 140-175 $\mu \mathrm{m}$ de largo en la región media (fig. 1C), ápices puntiagudos con una célula apical prominente (fig. 1D); todas las células de segundo orden producen células de tercer orden, no hay presencia de células intercalares (fig. 2) (organización apical tipo 1, Wynne, Price y Ballantine, 1989) que forman hileras de células hexagonales (fig. 3A) que se extienden hacia el margen del talo; células cerca del margen isodiamétricas (fig. 3A). Los talos están unidos al sustrato por pequeños discos de fijación o almohadillas y las hojas por rizoides marginales
A

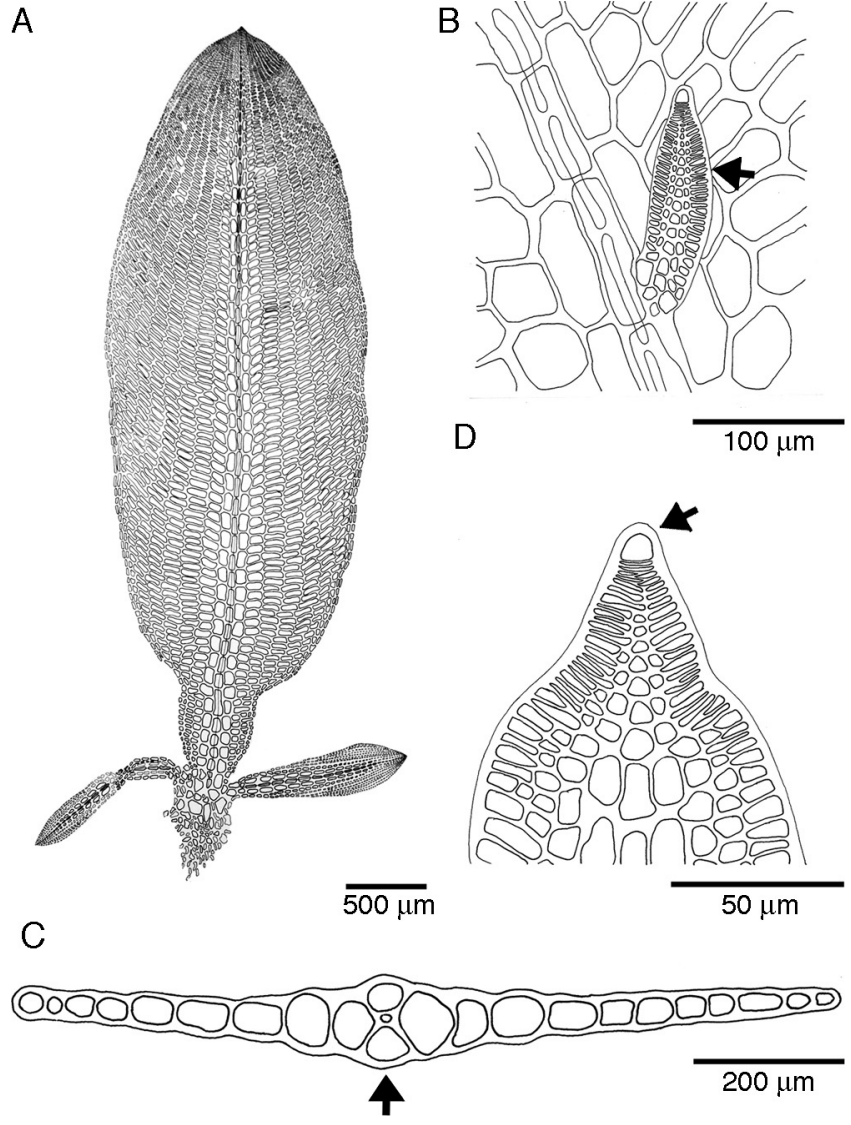

Figura 1. Hypoglossum hypoglossoides. A) aspecto general de la planta; B) detalle de la formación endógena de una rama joven naciendo del eje axial (flecha); C) corte transversal de la hoja media ecorticada, monostromática, en el centro célula axial y en los laterales células pericentrales (flecha); D) región apical con una célula prominente (flecha).

multicelulares (fig. 3A). De la fila del segundo orden se originan tetrasporangios esféricos (fig. 3B), 40-60 $\mu \mathrm{m}$ de diámetro, en soros elongados $(275-374 \times 760-856 \mu \mathrm{m})$ a lo largo de ambos lados del eje central y situados en la parte media de la hoja (fig. 3 C). Se observa especie inconspicua, epífita, donde inicia el casco del barco a $8 \mathrm{~m}$ de profundidad, que crece sobre las hojas de Dictyota friabilis Setchell, Jania pumila J. V. Lamouroux y Lobophora variegata (J. V. Lamouroux) Womersley ex E. C. Oliveira.

Hypoglossum hypoglossoides presenta una amplia distribución en el litoral del Atlántico occidental, en el hemisferio Norte: Bermudas (Collins y Hervey, 1917), Carolina del Norte (Schneider y Searles, 1991), Georgia (Searles, 1987), costa este de Florida (Hanisak y Blair, 1988, ejemplar a $42 \mathrm{~m}$ de profundidad), Belice (Norris y Bucher, 1982), Cuba (Martínez-Daranas, Díaz y Esquivel, 2002), Antillas Mayores (Littler y Littler, 2000), Puerto Rico (Wynne y Ballantine, 1986), Colombia (archipiélago de San Andrés, Díaz-Pulido y Díaz-Ruíz, 2003) y Venezuela (García, 2006); y en el hemisferio Sur: Brasil (Isla del Francés, Oliveira-Filho, 1969; Isla de Arvoredo, Santa Catarina, Horta et al., 2003).

Hypoglossum hypoglossoides se ha confundido con $\mathrm{H}$. tenuifolium, única especie del género registrada para México, en 


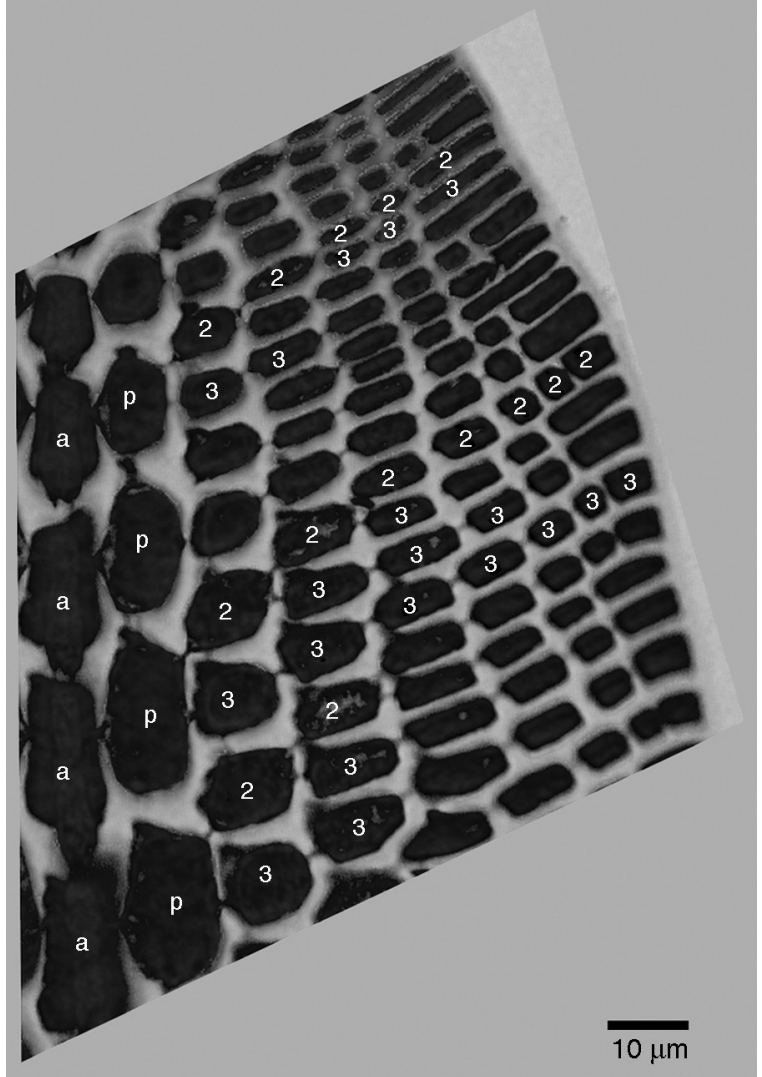

Figura 2. Organización de la región distal; hileras de células de segundo y de tercer orden. a: célula axial; p: célula pericentral.
Puerto Morelos, Quintana Roo (Dreckmann, Stout y SentíesGranados, 1996). No obstante, estas especies se diferencian en que $H$. hypoglossoides es de tamaño pequeño (hasta $3 \mathrm{~cm}$ de alto), postrado y con todas sus células de las filas de segundo orden que llevan a filas del tercer orden (fig. 2) (Kylin, 1923, 1924), mientras que en $H$. tenuifolium las hileras del tercer orden no provienen de las hileras del segundo orden y presentan láminas más grandes (hasta de $10 \mathrm{~cm}$ de alto) y erectas. Otras características que sirven para separar estas 2 especies son que $H$. hypoglossoides presenta un ápice puntiagudo y prominente (fig. 1D), mientras que en $H$. tenuifolium el ápice es hundido y romo (Wynne y Ballantine, 1986). En H. hypoglossoides los tetrasporangios se distribuyen azarosamente dentro de soros elongados que se forman a ambos lados del eje central (fig. 3B, C) (Kylin, 1924), mientras que en H. tenuifolium los trasporangios están distribuidos en hileras lineales en soros elongados a lo largo del eje central (Børgesen, 1919). En ambas especies las ramas surgen de forma endógena a partir de células axiales primarias y emergen de la línea media (fig. 1B).

Otras especies que se encuentran en la costa occidental tropical y subtropical, pero ausentes en la región mexicana del golfo de México (cf. Guiry y Guiry, 2015, para distribución geográfica), son: Hypoglossum anomalum (Caribe, América del Sur) se diferencia de $H$. hypoglossoides por presentar ramas que se producen en pares opuestos, que emergen de la línea media y en $H$. involvens (América del Norte, Caribe) los ápices de las hojas están fuertemente curvados (Wynne y Ballantine, 1986); en H. rhizophorum (América del Norte, Bermuda, Caribe) presenta una complejo sistema rizoidal característico de la especie

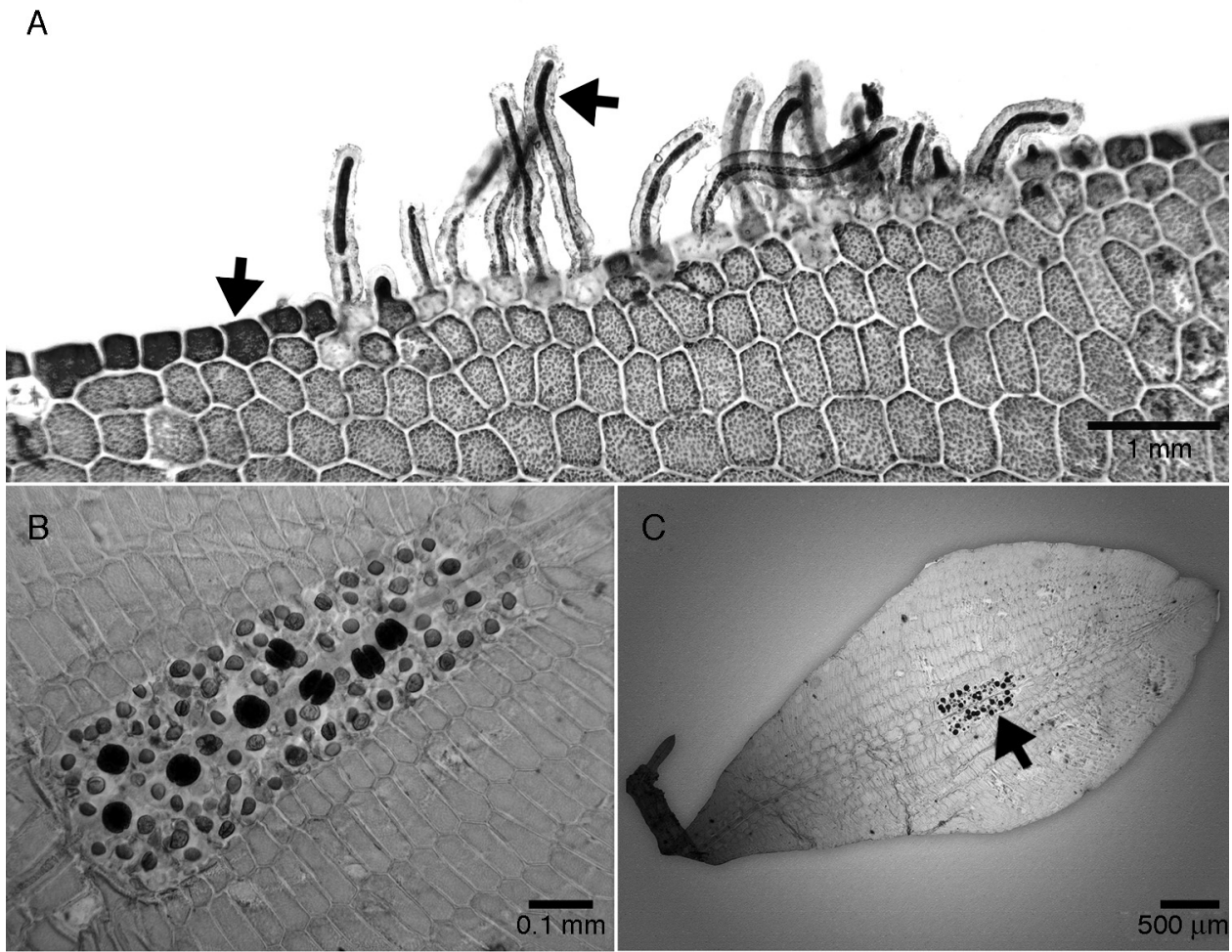

Figura 3. Hypoglossum hypoglossoides. A) región marginal con células isodiamétricas ecorticadas y rizoides marginales multicelulares (flechas); B) detalle del soro tetrasporangial con arreglo irregular de tetrasporangios; C) soro tetrasporangial en la región media de la hoja (flecha). 
y ausente en $H$. hypoglossoides (Ballantine y Wynne, 1988); H. simulans (Caribe) presenta una organización apical de tipo 2 , es decir, sólo algunas células del tercer orden son originadas de la fila de células del segundo orden, la especie en estudio presenta organización tipo 1 (Wynne et al., 1989); H. subsimplex (América del Norte, Bermuda, Caribe) presenta un arreglo diagonal de los tetrasporangios (forma "chevron"), un soro largo, continuo y situado distalmente (Wynne, 1994); H. caloglossoides (Bermuda, Caribe) presenta constricciones de los ejes decumbentes con un patrón de nodos e internodos (Wynne, 1994); $H$. barbatum (Bermuda) diverge de H. hypoglossoides por ser de hábito decumbente y presentar ramillas conspicuas en el segmento basal con abundantes rizoides en el ápice (Wynne et al., 1989).

Este registro nuevo complementa la distribución geográfica de una especie con afinidades a regiones cálido-templadas (rango de tolerancia de $15-30^{\circ} \mathrm{C}$, cf. Horta et al., 2003); de esta manera, $H$. hypoglossoides de Veracruz constituye un nuevo registro para México y pone de manifiesto la importancia de trabajar los pecios, sustratos artificiales con condiciones ambientales particulares, que tienen largo tiempo en el fondo marino, con el fin de complementar el inventario biótico del bentos veracruzano.

En el contexto del enfoque taxonómico moderno, la identificación morfológica presentada en este estudio tiene que ser confirmada en un futuro próximo por la comparación de secuencias de ADN para conocer que se trata de la misma especie en todo el intervalo distribucional americano, ya que podría haber especies crípticas. Se dispone de pocos estudios publicados sobre datos moleculares de la especie y se refieren a organismos de la región europea (Países Bajos, Choi, Kraft, Lee y Saunders, 2002; Lin et al., 2001). Esta observación es interesante, ya que será necesario compararlos con los ejemplares americanos y deslindar provincias geográficas (Europa, golfo de México y Caribe, Garduño-Solórzano, Godínez-Ortega y Ortega, 2005). Lin et al. (2001) señalan que $H$. hypoglossoides se encuentra dentro del grupo Hypoglossum con ausencia de células intercalares en todas las filas de células y células de tercer orden que logran llegar al margen de la fronda. Molecularmente esta especie entra en la tribu Hypoglosseae y está bien soportada para tener como tribus hermanas a Delesserieae y Grinnellieae. Choi et al. (2002) reafirman molecularmente que Hypoglossum se encuentra dentro de la familia Delesseriaceae, de origen polifilético y separado del complejo Dasyaceae/Delesseriaceae/Rhodomelaceae. La historia de vida de Hypoglossum es del tipo Polysiphonia (Ballantine y Wynne, 1988); sin embargo, este ciclo de vida no puede completarse por la carencia de las plantas gametangiales (masculinas y femeninas) en la región de estudio. Probablemente $H$. hypoglossoides es perenne, tal como lo documenta Díaz-Tapia y Bárbara (2014) para la península Ibérica. En la zona de estudio se encontró en 2 estaciones climáticas y oceanográficas contrastantes: secas (mayo) y nortes (febrero, marzo).

La investigación ficológica en el siglo XXI se ha caracterizado por el hallazgo de numerosos registros nuevos para México (Wynne, 2011), lo que indica que se están realizando mayores exploraciones en regiones poco investigadas en México que contribuyen al mejor conocimiento de la biota algal. En este estudio se explora por primera vez el Arrecife Anegada de Afuera y donde un barco crea un arrecife artificial rico en flora con afinidades a regiones mixtas. En el SAV todavía existen arrecifes por explorar: Anegadilla, Aviso, Cabezo, Chopas, El Rizo, Polo, Tierra Nueva y Topetillo (Galicia-García y Morales-García, 2007) y también es probable que en estas áreas se encuentren nuevos registros o especies de algas. Lo antes expuesto crea la necesidad de realizar exploraciones para buscar a Hypoglossum en diferentes regiones y sustratos del SAV para incrementar el conocimiento sobre su presencia en el golfo de México.

Se agradece al Instituto de Biología de la UNAM el apoyo para efectuar los trabajos de campo y a la Universidad Veracruzana el soporte financiero para la investigación. También agradecemos a Susana Guzmán la asesoría brindada en la microscopía estereoscópica digital, la reprografía de la figura 1A a Carmen Loyola y la realización de los dibujos a línea al Maestro Ricardo Manzanarez. Este trabajo se hizo en el marco de la Red para el Análisis y Síntesis de la Zona Costera Veracruzana, golfo de México (RASZCOV), apoyada por el Programa para el Desarrollo Profesional Docente (PRODEP-SEP).

\section{Referencias}

Ballantine, D. L. y Wynne, M. J. (1988). The life history and development of Hypoglossum rhizophorum (Delesseriaceae, Rhodophyta) in culture, a new deep-water species from the Caribbean. Journal of Phycology, 24, 8-12.

Børgesen, F. (1919). The marine algae of the Danish West Indies. Part 3. Rhodophyceae (5). Dansk Botanisk Arkiv, 3, 305-368.

Choi, H. G., Kraft, G. T., Lee, I. K. y Saunders, G. W. (2002). Phylogenetic analyses of anatomical and nuclear SSU rDNA sequence data indicate that the Dasyaceae and Delesseriaceae (Ceramiales Rhodophyta) are polyphyletic. European Journal of Phycology, 37, 551-569.

Collins, F. S. y Hervey, A. B. (1917). The algae of Bermuda. Proceedings of the American Academy of Arts and Sciences, 53, 1-195.

Díaz-Pulido, G. y Díaz-Ruíz, M. (2003). Diversity of benthic marine algae of the Colombian Atlantic. Biota Colombiana, 4, 203-246.

Díaz-Tapia, P. y Bárbara, I. (2014). Seaweeds from sand-covered rocks of the Atlantic Iberian Peninsula. Part 2. Palmariales, Ceramiales (excluding Rhodomelaceae), Gelidiales, Gigartinales, Plocamiales, Rhodymeniales and Scytothamnales. Cryptogamie Algologie, 35, 157-199.

Dreckmann, K. M., Stout, I. y Sentíes-Granados, A. (1996). Lista actualizada de las algas marinas bentónicas de Puerto Morelos, Quintana Roo Caribe Mexicano. Polibotánica, 3, 1-17.

Galicia-García, C. y Morales-García, A. (2007). Investigaciones sobre macroalgas realizadas en el Sistema Arrecifal Veracruzano. En A. Granados-Barba, L.G. Abarca-Arenas y J.M. Vargas-Hernández (Eds.), Investigaciones científicas en el Sistema Arrecifal Veracruzano (pp. 141-160). Campeche: Universidad Autónoma de Campeche.

García, M. (2006). Presencia de Hypoglossum hypoglossoides (Stackh.) Collins \& Herv. (Ceramiales Rhodophyta) en la costa venezolana. Acta Botánica Venezuelica, 29, 165-170.

Garduño-Solórzano, G., Godínez-Ortega, J. L. y Ortega, M. M. (2005). Distribución geográfica y afinidad por el sustrato de las algas verdes (Chlorophyceae) bénticas de las costas mexicanas del golfo de México y mar Caribe. Boletín de la Sociedad Botánica de México, 76, 61-78.

Guiry, M. D. y Guiry, G. M. (2015). AlgaeBase.World-wide electronic publication, National University of Ireland, Galway.Recuperado el 5 de febrero de 2015 de: http://www.algaebase.org.

Hanisak, M. D. y Blair, S. M. (1988). The deep-water macroalgal community of the East Florida continental shelf (USA). Helgoländer Meeresunters, 42, $133-163$.

Hayasaka-Ramírez, S. y Ortiz-Lozano, L. (2014). Anthropogenic pressure indicators associated with vessel groundings on coral reefs in a marine protected area. Ciencias Marinas, 40, 237-249. 
Horta, P., Yokoya, N., Guimarães, S., Bacci, D. y Oliveira, E. (2003). Morphology, reproduction and development of Hypoglossum hypoglossoides (Stackhouse) Collins \& Hervey (Ceramiales, Rhodophyta) from the South and Southeastern Brazilian coast. Revista Brasileira de Botânica, 26, 453-460.

Kylin, H. (1923). Studienuber die entwicklungsgeschichte der florideen Kungl. Svenska Vetenskapsakademiens Handlingar, 63, 1-138.

Kylin, H. (1924). Studien über die Delesseriaceen. Lunds Universitets Årsskrift, Ny Följd. Andra Afdelningen, 20, 1-111.

Lin, S. M., Fredericq, S. y Hommersand, M. H. (2001). Systematics of the Delesseriaceae (Ceramiales, Rhodophyta) based on large-subunit rDNA and rbcL sequences, including the Phycodryoideae, subfamily nov. Journal of Phycology, 37, 881-899.

Littler, D. S. y Littler, M. M. (2000). Caribbean reef plants: an identification guide to the reef plants of the Caribbean, Bahamas, Florida and Gulf of Mexico. Washington D.C.: Offshore Graphics Inc.

Martínez-Daranas, B., Díaz, J. y Esquivel, M. (2002). Nuevos registros de algas marinas cubanas (11). Avicennia, 15, 147-149.

Norris, J. N. y Bucher, K. E. (1982). Marine algae and seagrasses from Carrie Bow Cay, Belize. Smithsonian Contribution Marine Science, 12, 167-223.

Oliveira-Filho, E. C. (1969). Algas marinhas do sul do estado do Espirito Santo (Brasil). I.- Ceramiales. Boletim da Faculdade de Filosofia, Ciências e Letras da Universidade de São Paulo, Série Botânica, 26, 1-297.

Ortega, M. M., Godínez, J. L. y Garduño-Solórzano, G. (2001). Catálogo de las costas mexicanas del golfo de México y mar Caribe. México, D.F.: Instituto de Biología, UNAM.

Salas-Pérez, J. J. y Granados-Barba, A. (2008). Oceanographic characterization of the Veracruz Reef System. Atmósfera, 21, 281-301.
Schneider, C. W. y Searles, R. B. (1991). Seaweeds of the Southeastern United States: cape hatteras to cape Canaveral. Durham y London: Duke University Press.

Searles, R. B. (1987). Phenology and floristics of seaweeds from the offshore waters of Georgia. Northeast Gulf Science, 9, 99-108.

Tsuda, R.T. y Abbott, I. A. (1985). Collection, handling, preservation, and logistics.En M.M. Littler y D.S. Littler (Eds.), Handbook of phycological methods. Ecological field methods: macroalgae (pp. 67-86). Cambridge: Cambridge University Press.

Valadez-Rocha, V. y Ortiz-Lozano, L. (2013). Spatial and temporal effects of port facilities expansion on the surface area of shallow coral reefs. Environmental Management, 52, 250-260.

Womersley, H. B. S. y Shepley, E. A. (1982). Southern Australian species of Hypoglossum (Deleseriaceae Rhodophyta). Australian Journal of Botany, 30, 321-346.

Wynne, M. J. (1984). The correct name for the type of Hypoglossum Kützing (Delesseriaceae Rhodophyta). Taxon, 33, 56-58.

Wynne, M. J. (1994). The description of Hypoglossum subsimplex sp. nov. (Delesseriaceae, Rhodophyta) from the Florida Keys, Gulf of Mexico. Cryptogamie Algologie, 15, 253-262.

Wynne, M. J. (2011). A checklist of benthic marine algae of the tropical and subtropical western Atlantic: third revision. Nova Hedwigia Beiheft, 140, $1-166$.

Wynne, M. J. y Ballantine, D. L. (1986). The genus Hypoglossum Kützing (Delesseriaceae, Rhodophyta) in the tropical Western Atlantic, including $H$. anomalum sp. nov. Journal of Phycology, 22, 185-193.

Wynne, M. J., Price, I. R. y Ballantine, D. L. (1989). Distinctions between Hypoglossum barbatum Okamura, H. minimum Yamada and $H$. simulans sp. nov. (Delesseriaceae Rhodophyta). Phycologia, 28, 28-38. 